\title{
GnRH analogs do not protect ovaries from chemotherapy-induced ultrastructural injury in Hodgkin's lymphoma patients
}

\author{
Markus Nitzschke $\cdot$ Juliane Raddatz $\cdot$ \\ Michael K. Bohlmann · Petra Stute · \\ Thomas Strowitzki $\cdot$ Michael von Wolff
}

Received: 31 August 2009 / Accepted: 19 November 2009 / Published online: 6 December 2009

(C) Springer-Verlag 2009

\begin{abstract}
Purpose To determine the protective effect of gonadotropinreleasing hormone analogs ( $\mathrm{GnRHa}$ ) using several ultrasound and endocrine markers to detect ultrastructural ovarian damage in Hodgkin's lymphoma patients.

Methods Ten patients who had been treated for Hodgkin's lymphoma and had received GnRHa to protect ovarian function were matched with patients at similar age, who had received the same chemotherapy regimens without GnRHa. In addition, ten healthy women at the same age were matched to the study groups to compare ovarian markers. Blood samples were drawn to determine antiMüllerian hormone, Inhibin B, follicle-stimulating hormone and transvaginal ultrasound scans were performed to determine antral follicle count and ovarian volume. All women were asked about their menstrual cycle pattern.

Results No difference was found when comparing the ovarian parameters of both study groups. Compared with
\end{abstract}

M. Nitzschke and J. Raddatz contributed equally to this work.

M. Nitzschke $\cdot$ P. Stute $\cdot$ M. von Wolff $(\varangle)$

Department of Gynecological Endocrinology

and Reproductive Medicine, Woman's University

Hospital of Bern, Effingerstrasse 102, 3010 Bern, Switzerland

e-mail: Michael.vonWolff@insel.ch

J. Raddatz $\cdot$ T. Strowitzki

Department of Gynecological Endocrinology

and Reproductive Medicine, Woman's University

Hospital of Heidelberg, Heidelberg, Germany

M. K. Bohlmann

Department of Obstetrics and Gynecology,

University Hospital of Schleswig-Holstein,

Campus Luebeck, Luebeck, Germany healthy women, all ovarian parameters in the Hodgkin's lymphoma patients were significantly different.

Conclusions The results of this study demonstrate ultrastructural ovarian damage in Hodgkin's lymphoma patients irrespective of GnRHa co-treatment. These findings do not support previous studies, showing GnRHa to protect ovarian function.

Keywords GnRH analogs · Chemotherapy · Ovarian reserve $\cdot$ Fertility preservation $\cdot$ Hodgkin's lymphoma . Cancer

\section{Introduction}

Ovarian failure followed by amenorrhea and infertility is a common side effect of cytotoxic treatments for malignant disease in women [1]. These treatments cause depletion of the primordial follicle pool in a drug- and dose-dependent manner [2]. To sustain fertility in young women undergoing cytotoxic therapies, fertility preservation strategies have been placed into the focus of interest of health professionals and patients.

Among the currently discussed fertility preserving strategies, the concept of administering a non-invasive medical co-treatment such as gonadotropin-releasing hormone analogs (GnRHa) seems very attractive [3]. Glode et al. [4] using a murine model, tested this hypothesis for the first time almost three decades ago. They concluded that GnRHa appeared to protect male mice from the gonadal damage normally caused by cyclophosphamide, whereas several investigators have demonstrated that GnRHa can inhibit chemotherapy-induced ovarian follicular depletion in nonhuman species, uncertainty remains regarding human application [5]. 
Several studies have already tested the efficacy of $\mathrm{GnRHa}$ in humans. As described in three recent meta-analyses [6-8], the published clinical studies only provide evidence but still do not prove statistically that GnRHa co-treatment may reduce gonadal toxicity. This is due to the retrospective and non-randomized nature of most of the studies. A recent prospective randomized controlled trial found that the administration of GnRHa before and during chemotherapy for breast cancer could preserve post treatment ovarian function in young women [9].

However, more studies are needed. One reason is that a biological plausibility for gonadal protection by GnRHa in humans is still lacking [5] and another reason is that most studies only describe the prevalence of amenorrhea, even though depletion of primordial follicles may occur despite maintenance of regular menstrual cycles $[10,11]$. The amount of surviving primordial follicles after cytotoxic treatments can only be quantified in vivo using several distinct hormonal and ultrasound-based surrogates [12].

Therefore we set up this small, but carefully designed trial with patients who were exactly matched for age and treatment. A broad set of ultrasound parameters (antral follicle count (AFC), ovarian volume) and endocrine markers (follicle-stimulating hormone (FSH), anti-Müllerian hormone (AMH), Inhibin B) were used to measure ovarian reserve more precisely than simple prevalence of amenorrhea could do.

\section{Materials and methods}

This case-control study was performed at the Department of Oncology together with the Department of Endocrinology and Reproductive Medicine at the University Hospital of Heidelberg (Germany) in cooperation with the German Hodgkin Study Group (http://www.ghsg.org). The local ethics review committee approved the study protocol and a written informed consent was obtained from all patients.

Patient population

Ten patients who had received chemotherapy and radiotherapy for Hodgkin's lymphoma between 2001 and 2007 had been co-treated with GnRHa (leuprorelin $3.57 \mathrm{mg} \mathrm{s.c}$ or goserelin $3.6 \mathrm{mg}$ s.c. monthly before and during chemotherapy) to protect ovarian function (Group A).

In cooperation with the German Hodgkin Study Group, we localized a total of 31 patients who had also been treated for Hodgkin's lymphoma in nearby University Hospitals during the same period of time, but had not received GnRHa. These patients were carefully matched to our study group for age and a similar chemotherapy regimen (Group B). Ten additional healthy women, who were treated in our department of reproductive medicine for severe male factor infertility, were matched to our study groups by age to compare the ovarian markers (Table 1).

The following chemotherapies were used: Four patients received two cycles of ABVD (Adriamycin, Bleomycin, Vinblasin, Dacarbacin), eight patients received four cycles of ABVD, six patients received two cycles of OPPA (Oncovin, Procarbacin, Prednison, Adriamycin) and two cycles of COPP (Cyclophosphamid, Oncovin, Procarbacin, Prednison) and two patients received eight cycles of BEACOPP-14 (Bleomycin, Etoposid, Adriamycin, Cyclophosphamid, Oncovin, Procarbacin, Prednison). All radiotherapies were supradiaphragmal. At the time the study was performed, all patients had completed their treatment cycles at least 6 months before.

Study protocol

All participating women were seen twice within a 1- to 3month period. Except the two patients with amenorrhea, all women were seen at the second to fifth day of the cycle. No woman was taking oral contraception (OC) or hormone replacement therapy (HRT) during the study period. Blood samples were drawn to determine $\mathrm{AMH}$, Inhibin B and FSH in the serum and transvaginal ultrasound scans were performed to determine AFC and ovarian volume. All women were asked about their menstrual cycle pattern.

Treatment of the samples

Sera were obtained after centrifugation and stored at $-80^{\circ} \mathrm{C}$ in five aliquots of $2 \mathrm{~mL}$ in standard Eppendorf tubes. The samples were centrifuged for $10 \mathrm{~min}$ at 3,500 rpm. Concentrations of FSH were analyzed using commercially available immunoradiometric kits (Boehringer, Mannheim, Germany) and analyzed on the Elecsys immunoassay analyzer (Roche Molecular Biochemicals, Mannheim, Germany).

AMH was assayed using an ultra sensitive ELISA (AMH/MIS active coated well ELISA, DSL-10-14400, Diagnostic System Laboratories, DSL, TX, USA). The assay range was $0.025-15 \mathrm{ng} / \mathrm{mL}$, and the functional sensitivity $0.017 \mathrm{ng} / \mathrm{mL}$. Intra-assay and inter-assay coefficients of variance were 3.4 and $6.5 \%$, respectively (conversion factor to $\mathrm{pmol} / \mathrm{L}=\mathrm{ng} / \mathrm{mL} \times 7.143$ ). Inhibin $B$ was quantified with the Inhibin B active coated well ELISA, DSL-10-84100 (Diagnostic System Laboratories, DSL, TX, USA). The assay range was $10-531 \mathrm{pg} / \mathrm{mL}$, and the functional sensitivity $7 \mathrm{pg} / \mathrm{mL}$. Intra-assay and inter-assay coefficients of variance 
Table 1 Comparison between Hodgkin's lymphoma patients with (+) GnRHa and without (-) GnRHa co-treatment and healthy women

\begin{tabular}{|c|c|c|c|c|c|c|c|c|c|c|}
\hline & Group & Pat. ID & Age & $\begin{array}{l}\text { Therapy } \\
\text { (chemo-/IF-RT) }\end{array}$ & Mens & $\begin{array}{l}\text { FSH } \\
(\mathrm{U} / \mathrm{L})\end{array}$ & $\begin{array}{l}\text { AMH } \\
(\mathrm{ng} / \mathrm{mL})\end{array}$ & $\begin{array}{l}\text { Inhibin B } \\
\text { (pg/mL) }\end{array}$ & AFC & $\begin{array}{l}\text { Ov-vol } \\
\left(\mathrm{cm}^{3}\right)\end{array}$ \\
\hline \multirow[t]{3}{*}{1} & Healthy & U.S & 28 & - & reg. & 9.2 & 3.2 & 73.3 & 11 & 9.3 \\
\hline & Hod + GnRHa & C.G & 26 & $2 \times \mathrm{ABVD} / \mathrm{s}$ & reg. & 6.4 & 4.3 & 23.2 & 12 & 13.6 \\
\hline & Hod - GnRHa & J.F & 30 & $2 \times \mathrm{ABVD} / \mathrm{s}$ & reg. & 6.7 & 2.4 & 7.8 & 11 & 11.8 \\
\hline \multirow[t]{3}{*}{2} & Healthy & D.H & 20 & - & reg. & 5.3 & 5.4 & 79.5 & 19 & 12.6 \\
\hline & $\mathrm{Hod}+\mathrm{GnRHa}$ & I.G & 20 & $2 \times \mathrm{ABVD} / \mathrm{s}$ & reg. & 8.1 & 1.9 & 23.6 & 0 & 3.1 \\
\hline & Hod - GnRHa & I.H & 23 & $2 \times \mathrm{ABVD} / \mathrm{s}$ & reg. & 6.3 & 1.1 & 90.9 & 6 & 4.7 \\
\hline \multirow[t]{3}{*}{3} & Healthy & J.S & 21 & - & reg. & 3.3 & 5.9 & 76.5 & 10 & 9.3 \\
\hline & Hod + GnRHa & M.Mz & 20 & $4 \times \mathrm{ABVD} / \mathrm{s}$ & reg. & 5.9 & 1.5 & 11.5 & 5 & 7.1 \\
\hline & Hod - GnRHa & D.W & 23 & $4 \times \mathrm{ABVD} / \mathrm{s}$ & reg. & 5.5 & 3.0 & 8.5 & 16 & 8.0 \\
\hline \multirow[t]{3}{*}{4} & Healthy & N.B. & 25 & - & reg. & 6.9 & 4.1 & 79.5 & 12 & 7.5 \\
\hline & Hod + GnRHa & S.B. & 25 & $4 \times \mathrm{ABVD} / \mathrm{s}$ & reg. & 4.4 & 4.1 & 41.3 & 18 & 13.6 \\
\hline & Hod - GnRHa & S.O. & 26 & $4 \times \mathrm{ABVD} / \mathrm{s}$ & reg. & 3.7 & 1.5 & 5.4 & 9 & 5.9 \\
\hline \multirow[t]{3}{*}{5} & Healthy & J.R. & 28 & - & reg. & 5.6 & 3.7 & 74.2 & 14 & 6.6 \\
\hline & Hod + GnRHa & K.H & 29 & $4 \times \mathrm{ABVD} / \mathrm{s}$ & reg. & 2.4 & 1.6 & 2.1 & 0 & 6.7 \\
\hline & Hod - GnRHa & C.S. & 27 & $4 \times \mathrm{ABVD} / \mathrm{s}$ & reg. & 5.3 & 1.3 & 1.3 & 6 & 4.8 \\
\hline \multirow[t]{3}{*}{6} & Healthy & S.R. & 30 & - & reg. & 6.9 & 3.3 & 94.6 & 15 & 15.3 \\
\hline & Hod + GnRHa & N.K. & 30 & $4 \times \mathrm{ABVD} / \mathrm{s}$ & reg. & 6.0 & 1.5 & 18.6 & 3 & 5.7 \\
\hline & Hod - GnRHa & S.M. & 33 & $4 \times \mathrm{ABVD} / \mathrm{s}$ & reg. & 6.3 & 3.4 & 74.1 & 10 & 5.1 \\
\hline \multirow[t]{3}{*}{7} & Healthy & F.C. & 18 & - & reg. & 5.7 & 2.4 & 61.8 & 8 & 12.7 \\
\hline & Hod + GnRHa & L.K. & 17 & $2 \times \mathrm{OPPA}+2 \times \mathrm{COPP} / \mathrm{s}$ & reg. & 5.6 & 0.5 & 11.3 & 6 & 13.3 \\
\hline & Hod - GnRHa & C.B. & 17 & $2 \times \mathrm{OPPA}+2 \times \mathrm{COPP} / \mathrm{s}$ & reg. & 6.5 & 0.2 & 95.4 & 0 & 2.4 \\
\hline \multirow[t]{3}{*}{8} & Healthy & T.B. & 19 & - & reg. & 4.3 & 5.5 & 38.0 & 11 & 7.6 \\
\hline & Hod + GnRHa & E.H. & 19 & $2 \times \mathrm{OPPA}+2 \times \mathrm{COPP} / \mathrm{s}$ & reg. & 10.0 & 0.0 & 0.0 & 4 & 1.6 \\
\hline & Hod - GnRHa & S.H. & 27 & $2 \times \mathrm{OPPA}+2 \times \mathrm{COPP} / \mathrm{s}$ & reg. & 7.0 & 0.3 & 8.7 & 3 & 3.9 \\
\hline \multirow[t]{3}{*}{9} & Healthy & J.J. & 21 & - & reg. & 4.1 & 6.7 & 82.5 & 13 & 16.3 \\
\hline & Hod + GnRHa & K.F. & 20 & $2 \times \mathrm{OPPA}+2 \times \mathrm{COPP} / \mathrm{s}$ & reg. & 26.2 & 0.2 & 11.2 & 8 & 8.2 \\
\hline & Hod - GnRHa & A.S1. & 22 & $2 \times \mathrm{OPPA}+2 \times \mathrm{COPP} / \mathrm{s}$ & reg. & 6.2 & 0.7 & 40.0 & 6 & 4.6 \\
\hline \multirow[t]{3}{*}{10} & Healthy & M.E. & 34 & - & reg. & 2.8 & 4.0 & 96.4 & 14 & 7.5 \\
\hline & Hod + GnRHa & N.S. & 33 & $8 \times$ BEACOPP-14/s & am. & 71.7 & 0.0 & 0.0 & 0 & 1.6 \\
\hline & Hod - GnRHa & E.P. & 35 & $8 \times$ BEACOPP-14/s & am. & 91.7 & 0.0 & 0.0 & 0 & 1.9 \\
\hline
\end{tabular}

Age age when study was performed, $R T$ radio therapy, $s$ supradiaphragmal radio therapy, $I F$ involved field radio therapy, Mens menstrual cycle, reg. regular, am. amenorrhea, $A M H$ anti-Muellerian hormone, $A F C$ antral follicle count, $O v$-vol ovarian volume

were 4.6 and $6.7 \%$, respectively. The manufacturer's protocol was followed in both cases. All assays were done in duplicate.

\section{Ultrasound scans}

The ultrasound scans were performed via transvaginal probe by the same investigator. The examining person was blinded concerning hormonal status and medical history of the patient. Antral follicles (2-12 mm) were counted on both ovaries [13] and video monitored to allow analysis by a second examiner. Ovarian volume was calculated using the prolate ellipsoid formula $L \times H \times$ $W \times 0.523$ ( $L=$ length in mid sagital, $H=$ height in mid sagital, $W=$ width in axial) [14]. The volume of both ovaries was added.

Outcome measures

The main outcome measures to compare the three study groups were: secondary amenorrhea, FSH (U/L), AMH $(\mathrm{ng} / \mathrm{mL})$, Inhibin B (pg/mL), AFC and ovarian volume $\left(\mathrm{cm}^{3}\right)$.

Statistical analysis

Mann-Whitney $U$ test was used as a non-parametric test to compare the parameters of ovarian reserve between the 
study groups. Statistical significance was presumed to be achieved for values of $P \leq 0.05$.

\section{Results}

Characteristics of trial participants

The mean age of patients treated for Hodgkin's lymphoma (Group A + B) was 25.1 [SD 5.2] years when the study was performed (range 17-35 years). There was no significant difference in the mean age between Groups A and B (23.9 [SD 5.1] and 26.3 [SD 5.1] years). Mean age of the group of healthy controls was 26.3 [SD 5.1] years (range 18-35 years). Again no significant difference was seen in the mean age between the study groups (Group $\mathrm{A}+\mathrm{B}$ ) and the healthy women. Both patients who had received BEACOPP-14 (one in each study group) experienced secondary amenorrhea.

The time interval between the end of the chemotherapy and the analysis of the ovarian parameters was 30.6 months [SD 7.2] for Group A (range 19-45 months) and 32.2 months [SD 20.4] for Group B (range 6-58 months). Patients were not matched for this time interval for the following reasons:

As the development of AMH producing secondary and tertiary follicles takes only around 3 months, ovarian function is sufficiently restored not later than 6 months after the end of chemotherapy. Thereafter, AMH concentrations remain stable in the same subject. The intra-individual variation of $\mathrm{AMH}$ is only $8 \%$ (coefficient of variation) if $\mathrm{AMH}$ is measured in the same subjects initially and again after 2 months according to an own unpublished study in 21 patients. For this reason and for the fact that other parameters such as age and the type of chemotherapy are far more important parameters to be matched, we accepted a limited variation in the time interval between the end of the chemotherapy and the AMH measurement.

Outcome of study groups

No difference was found when comparing the ovarian parameters in Hodgkin patients with (Group A) and without (Group B) GnRHa co-treatment. For FSH, the mean concentration in Group A was 14.7 U/L [SD 6.6] and in Group B 14.5 U/L [SD 0.9]. The mean concentration of AMH in Group A was $1.6 \mathrm{ng} / \mathrm{mL}$ [SD 1.4] and in Group B $1.4 \mathrm{ng} /$ $\mathrm{mL}$ [SD 1.1]. For Inhibin $\mathrm{B}$, the mean concentration in Group A was 14.3 pg/mL [SD 8.7] and in Group B 33.2 pg/ $\mathrm{mL}$ [SD 12.5]. Moreover, no significant difference was seen for the mean AFC (Group A: 5.6 follicles [SD 5.5] and Group B: 6.7 follicles [SD 4.7]) or the mean ovarian volume (Group A: $7.5 \mathrm{~cm}^{3}$ [SD 4.5] and Group B: $5.3 \mathrm{~cm}^{3}$ [SD 2.6]) in both groups (Fig. 1).

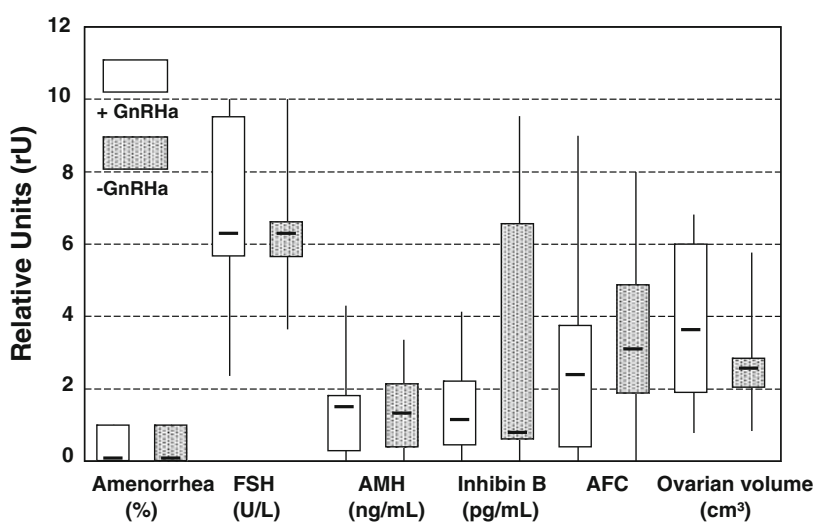

Fig. 1 Comparison of ovarian parameters in Hodgkin's lymphoma patients with (+) and without (-) GnRHa co-treatment

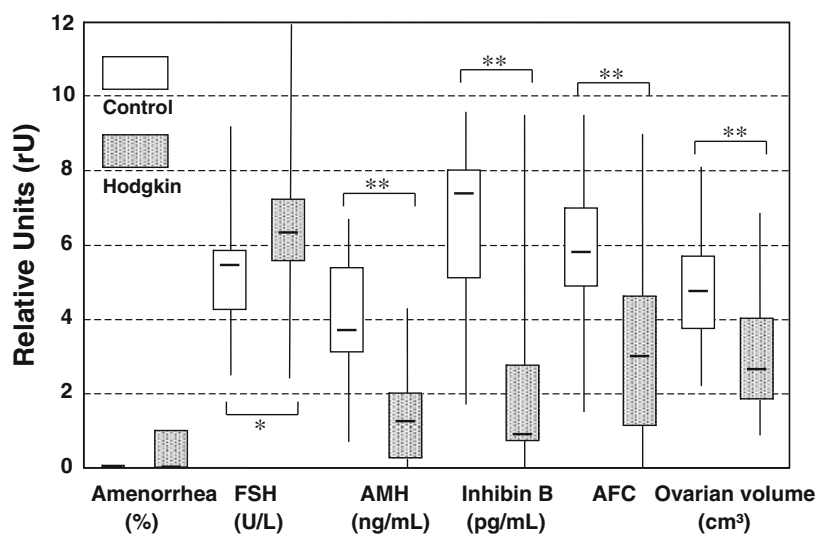

Fig. 2 Ovarian parameters in healthy women compared with those in Hodgkin's lymphoma patients of the same age $\left({ }^{*} P<0.05 ; * * P<0.01\right)$

Compared with the healthy women of the control group, all ovarian parameters in the Hodgkin's lymphoma patients (Group A + B) were significantly different. The mean concentration of FSH was significantly higher in patients after chemotherapy (14.6 U/L [SD 4.8] vs. 5.3 U/L [SD 1.8]). The mean concentration of AMH and Inhibin B was significantly lower in those women $(1.5 \mathrm{ng} / \mathrm{mL}$ [SD 1.3] vs. $3.9 \mathrm{ng} / \mathrm{mL}$ [SD 1.2] for AMH and $23.7 \mathrm{pg} / \mathrm{mL}$ [SD 18.6] vs. $64.9 \mathrm{pg} / \mathrm{mL}$ [SD 15.7] for Inhibin B). The AFC and the ovarian volume were significantly reduced in the Hodgkin's groups as well (6.2 follicles [SD 4.5] vs. 11.6 follicles [SD 2.9] for AFC and $6.4 \mathrm{~cm}^{3}$ [SD 3.8] versus $9.7 \mathrm{~cm}^{3}$ [SD 3.3] for ovarian volume). The $P$ value for FSH was $P<0.05$ and for all other parameters $P<0.01$ (Fig. 2).

\section{Discussion}

The concept of using GnRHa to prevent chemo- or radiotherapy induced gonadal damage was introduced by Glode et al. [4]. Since then, several animal studies suggested a 
protective role of GnRHa co-treatment against follicle loss during cytotoxic treatments. However, no benefit could be demonstrated against radiotherapy induced gonadal damage $[15,16]$. It has been highly debated whether human application of GnRHa would be safe and useful to preserve fertility and ovarian function in women treated for cancer [5-7].

In a recent prospective randomized study including 80 women receiving chemotherapy for breast cancer, GnRHa co-treatment was demonstrated to be effective in preserving ovarian function [9]. Patients were randomly assigned to receive chemotherapy combined with monthly injections of goserelin or chemotherapy alone. After 8 months of followup, $89.6 \%$ of the women in the study group (with GnRHa) resumed menses and $69.2 \%$ had spontaneous ovulation. In contrast only $33.3 \%$ of the patients in the control group (without GnRHa) resumed menses and $25.6 \%$ had spontaneous ovulation. These results were highly significant $(P<0.001)$. However, this study suffers from an important shortcoming: The control group had significantly higher mean serum FSH and Estradiol $\left(\mathrm{E}_{2}\right)$ levels even before start of the treatment. As both are important endocrine markers of ovarian reserve, a difference between the study groups could be, at least in a part, due to a lower initial ovarian reserve in the control group.

A second published prospective randomized study, including 30 men and 18 women receiving chemotherapy for Hodgkin's disease, revealed that GnRHa treatment seemed to be ineffective in preserving fertility as judged by sperm count and menstrual function [17]. Patients were randomly assigned to receive buserelin (20 men and 8 women) prior to and for the duration of cytotoxic chemotherapy. After 3 years of follow-up, all men in both, the study and control, groups became oligo/azoospermic, while four of the eight women treated with a GnRHa and six of the nine female controls became amenorrheic.

In a third randomized study, Sverrisdottir et al. [18] examined if goserelin as adjuvant treatment protects the ovaries from premature failure in premenopausal breast cancer patients. A total of 285 patients received CMF (cyclophosphamide, methotrexate and 5-fluorouracil). All patients were randomly assigned to receive 2 years of goserelin, goserelin plus tamoxifen, tamoxifen alone or no endocrine treatment. Endocrine treatment was given concomitant to chemotherapy. One year after completed CMF and endocrine therapy, $36 \%$ of the women in the goserelin group reported menses, compared to $7 \%$ in the goserelin plus tamoxifen group, $13 \%$ in the tamoxifen group and $10 \%$ of the controls.

Several other non-randomized studies in the past provided mixed results [6]. In addition to the lack of consistent support from clinical studies, there is currently no biological explanation for how GnRHa can protect ovarian reserve. Primordial follicles do not express FSH or GnRH receptors [5]. GnRHa, in essence, returns the hormonal milieu to prepubertal state. A clinical example for why gonadal suppression may not protect ovaries is the fact that prepubertal children receiving high-dose chemotherapy given before hematopoietic stem cell transplantation still suffer from ovarian failure [19]. Nevertheless, because younger patients have a larger ovarian reserve, they might tolerate some loss in the follicular pool, and immediate ovarian function might not be affected in the short term. All patients who receive high-dose gonadotoxic chemotherapy will eventually suffer from premature ovarian failure [20, 21].

In our study, a broad spectrum of five sensitive parameters was used to analyze ovarian damage after cytotoxic treatment more precisely than the simple determination of secondary amenorrhea could do.

Only the two patients who had received the BEACOPP14 regimen experienced secondary amenorrhea, whereas all other patients were still regularly menstruating. However, using sensitive parameters to compare ovarian reserve in Hodgkin's lymphoma patients and healthy women, we were able to demonstrate highly significant differences. This was also the case in those patients who had received the ABVD regimen, which was thought not to affect ovarian reserve. According to our results, chemotherapy for Hodgkin's disease can, therefore, be considered to potentially accelerate ovarian aging.

Furthermore, we could demonstrate ultrastructural ovarian damage in Hodgkin's lymphoma patients who were co-treated with GnRHa. The ovarian damage was, according to our parameters, identical to those patients who had not received GnRHa. We thus conclude that GnRHa did not seem to protect the ovaries from the cytotoxic effect of the chemotherapies in our setting.

Our findings are in concordance with the results of the only other study using sensitive ovarian parameters to measure the ovarian reserve after chemotherapy and GnRHa co-treatment. Giuseppe et al. [22] evaluated methods to assess the ovarian reserve in 29 women with Hodgkin's disease treated with chemotherapy. Furthermore, they examined the effect of GnRHa co-treatment on ovarian function. Ovarian reserve was studied by measuring the serum levels of FSH, LH, Inhibin B, AMH and AFC following treatment with or without GnRHa. They did not find a significant difference of all parameters measured following GnRHa treatment in comparison to the untreated control group.

Obviously the power of this study as well as of our study is limited due to the low number of analyzed patients. However, analysing previous studies in detail reveals that a huge number of patients are needed to draw distinct conclusions concerning the effect of GnRHa. The high number of different and heterogeneous chemotherapy regimes, the age 
dependency of individual ovarian reserve as well as the application of the rough parameter "amenorrhea" also limits the power of the previously published prospective randomized studies. Accurate matching of patients and controls in relation to chemotherapy and age as well as the application of several sensitive parameters to determine ovarian reserve does allow drawing a conclusion irrespective of the small number of treated patients in our study. It thereby contributes to the discussion about the efficiency of GnRHa regardless of its design.

We could also demonstrate that only the application of a broad set of sensitive parameters may properly reflect ovarian reserve. Therefore, these markers may be used to better quantify the damage of the ovaries following exposition to chemotherapies in future studies. Such analyses may not only require a prospective randomised design, but also the determination of several sensitive ovarian markers to measure the supposed protective effect of GnRHa on ultrastructural changes of ovarian function during cytotoxic treatment. Such studies will hopefully finally solve the still open question concerning the efficacy of GnRHa as a fertility preserving technique.

Acknowledgments We would like to thank the German Hodgkin Study Group for assistance (http://www.ghsg.org).

Conflict of interest statement None.

\section{References}

1. Meirow D, Nugent D (2001) The effects of radiotherapy and chemotherapy on female reproduction. Hum Reprod Update 7:535-543

2. Sonmezer M, Oktay K (2004) Fertility preservation in female patients. Hum Reprod Update 10:251-266

3. Blumenfeld Z, Avivi I, Ritter M, Rowe JM (1999) Preservation of fertility and ovarian function and minimizing chemotherapy-induced gonadotoxicity in young women. J Soc Gynecol Investig 6:229-239

4. Glode LM, Robinson J, Gould SF (1981) Protection from cyclophosphamide-induced testicular damage with an analogue of gonadotropin-releasing hormone. Lancet 1:1132-1134

5. Oktay K, Sönmezer M, Oktem O, Fox K, Emons G, Bang H (2007) Absence of conclusive evidence for the safety and efficacy of gonadotropin-releasing hormone analogue treatment in protecting against chemotherapy-induced gonadal injury. Oncologist 12:1055-1066

6. Blumenfeld Z, von Wolff M (2008) GnRH-analogues and oral contraceptives for fertility preservation in women during chemotherapy. Hum Reprod Update 14:543-552

7. Beck-Fruchter R, Weiss A, Shalev E (2008) GnRH agonist therapy as ovarian protectants in female patients undergoing chemotherapy: a review of the clinical data. Hum Reprod Update 14:541-542
8. Clowse ME, Behera MA, Anders CK, Copland S, Coffman CJ, Leppert PC, Bastian LA (2009) Ovarian preservation by GnRH agonists during chemotherapy: a meta-analysis. J Womens Health 18:311-319

9. Badawy A, Elnashar A, El-Ashry M, Shahat M (2009) Gonadotropin-releasing hormone agonists for prevention of chemotherapyinduced ovarian damage: prospective randomized study. Fertil Steril 91:694-697

10. Bath LE, Wallace WH, Shaw MP, Fitzpatrick C, Anderson RA (2003) Depletion of ovarian reserve in young women after treatment for cancer in childhood: detection by anti-Müllerian hormone, inhibin B and ovarian ultrasound. Hum Reprod 18:2368-2374

11. Larsen EC, Müller J, Rechnitzer C, Schmiegelow K, Andersen AN (2003) Diminished ovarian reserve in female childhood cancer survivors with regular menstrual cycles and basal FSH $<10 \mathrm{IU} / \mathrm{l}$. Hum Reprod 18:417-422

12. Anderson RA, Themmen AP, Al-Qahtani A, Groome NP, Cameron DA (2006) The effects of chemotherapy and long-term gonadotrophin suppression on the ovarian reserve in premenopausal women with breast cancer. Hum Reprod 21:2583-2592

13. Avril C (2006) Antral follicle count and oocyte quality. J Gynecol Obstet Biol Reprod 35:2S42-2S43

14. Pavlik EJ, DePriest PD, Gallion HH, Ueland FR, Reedy MB, Kryscio RJ et al (2000) Ovarian volume related to age. Gynecol Oncol 77:410-412

15. Ataya K, Pydyn E, Ramahi-Ataya A, Orton CG (1995) Is radiation-induced ovarian failure in rhesus monkeys preventable by luteinizing hormone-releasing hormone agonists?: preliminary observations. J Clin Endocrinol Metab 80:790-795

16. Ataya K, Rao LV, Lawrence E, Kimmel R (1995) Luteinizing hormone-releasing hormone agonist inhibits cyclophosphamideinduced ovarian follicular depletion in rhesus monkeys. Biol Reprod 52:365-372

17. Waxman JH, Ahmed R, Smith D, Wrigley PF, Gregory W, Shalet S et al (1987) Failure to preserve fertility in patients with Hodgkin's disease. Cancer Chemother Pharmacol 19:159-162

18. Sverrisdottir A, Nystedt M, Johansson H, Fornander T (2009) Adjuvant goserelin and ovarian preservation in chemotherapy treated patients with early breast cancer: results from a randomized trial. Breast Cancer Res Treat 117:561-567

19. Teinturier C, Hartmann O, Valteau-Couanet D, Benhamou E, Bougneres PF (1998) Ovarian function after autologous bone marrow transplantation in childhood: high-dose busulfan is a major cause of ovarian failure. Bone Marrow Transplant 22:989-994

20. Grigg AP, McLachlan R, Zaja J, Szer J (2000) Reproductive status in long-term bone marrow transplant survivors receiving busulfancyclophosphamide $(120 \mathrm{mg} / \mathrm{kg})$. Bone Marrow Transplant 26:1089-1095

21. Viviani S, Santoro A, Ragni G, Bonfante V, Bestetti O, Bonadonna G (1985) Gonadal toxicity after combination chemotherapy for Hodgkin's disease. Comparative results of MOPP vs ABVD. Eur J Cancer Clin Oncol 21:601-605

22. Giuseppe L, Attilio G, Edoardo DN, Loredana G, Cristina L, Vincenzo L (2007) Ovarian function after cancer treatment in young women affected by Hodgkin disease (HD). Hematology 12:141-147 